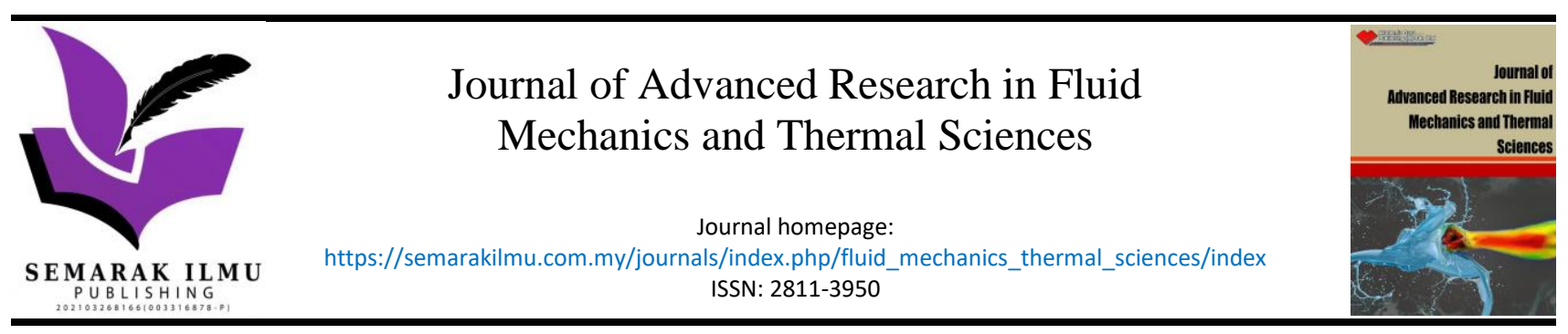

\title{
Stability Analysis of The Stagnation-Point Flow and Heat Transfer Over a Shrinking Sheet in Nanofluid in The Presence of MHD and Thermal Radiation
}

\author{
Nurul Syuhada Ismail ${ }^{1,}$, Yong Faezah Rahim², Norihan Md Arifin ${ }^{3}$, Roslinda Nazar ${ }^{4}$, Norfifah \\ Bachok $^{3}$ \\ 1 Centre for Pre-University Studies, Universiti Malaysia Sarawak, 94300, Kota Samarahan, Sarawak, Malaysia \\ Centre of Foundation Studies for Agriculture Science, Universiti Putra Malaysia, 43400 UPM Serdang, Selangor, Malaysia \\ 3 Department of Mathematics, Faculty of Science and Institute for Mathematical Research, Universiti Putra Malaysia, 43400 UPM Serdang, \\ Selangor, Malaysia \\ 4 School of Mathematical Sciences, Faculty of Science and Technology, Universiti Kebangsaan Malaysia, 43600UKM Bangi, Selangor, Malaysia
}

\section{ARTICLE INFO}

\section{Article history:}

Received 24 August 2021

Received in revised form 6 December 2021

Accepted 13 December 2021

Available online 26 January 2022

\section{Keywords:}

Nanofluid; shrinking sheet; stability analysis; stagnation point; thermal radiation

\section{ABSTRACT}

\begin{abstract}
Recent developments in the field of fluid dynamics have led to a new interest in stability analysis. The numerical solution obtained from the problems of the flow at the stagnation point, as well as the heat transfer with MHD and thermal radiation effects over a shrinking sheet, is used to carry out a stability analysis. The flow of this problem is considered in nanofluids and Buongiorno's model is employed. The boundary layer equation is obtained by reducing the governing equations to an ordinary differential equation. Partial differential equations are converted to ordinary differential equations using a suitable similarity transformation. The bvp4c simulation on Matlab is then used to solve ordinary differential equations. According to the numerical data, the dual solutions occur in a specific range of $\alpha$. The parameter $\alpha$ refers to the stretching/shrinking where shrinking (less than 0 ) is the main reason the dual solution exists. The stability analysis is presented graphically and in tabular form to prove that there are two solutions to the problem and only one of them is stable. As a result, our research shows that the solution is only stable in the first solution, but not in the second.
\end{abstract}

\section{Introduction}

Research on heat transfer problems is important for applications in industries and engineering, as reported by Oo et al., [1], Idris et al., [2], Jahan et al., [3], Pasha et al., [4] and Halim and Sidik [5]. However, if non-unique solutions occur in calculations, it is important to verify the stability of the solutions. There are some considerations that lead to the dual solution of some problems, such as in the unsteady case, mixed convection, moving surface and stretching or shrinking surface in boundary layer flow. So far, only this condition that affects the dual solution occurs in the literature review, and some existences occur only under certain conditions. For example, in certain cases, to gain the

\footnotetext{
* Corresponding author.

E-mail address: insyuhada@unimas.my
}

https://doi.org/10.37934/arfmts.91.2.96105 
dual solution in the stretching sheet, the presence of suction is needed, but a shrinking sheet can produce the dual solution without the presence of suction.

The literature review shows that Merkin [6] has been the first person to thoroughly investigate how stable the first solution is, and how unstable the second. This research has also been explored in prior studies by Merrill et al., [7], where the lowest eigenvalues for both the first and second branches have been plotted for the porous medium problem. This problem exists when there is a steady flow on a vertical surface near to a stagnation point. The research conducted by Harris et al., [8] for mixed convection problems has discussed the suggestions with detailed elaborations. By relaxing an appropriate boundary condition, the range of possible eigenvalues can be investigated. Only one condition will be relaxed, and usually, it is chosen from " $\eta \rightarrow \infty$ " condition. The replacement will be 1-order higher, to be placed under " $\eta=0$ " condition and will be equal to 1 . The suggestion of Harris et al., [8] has been used by the next researchers. Some of these papers can be seen in this regard, such as Roşca and Pop [9], Weidman et al., [10], Postelnicu and Pop [11], Ishak [12,13], and Ismail et al., [14-17].

Physically, the solution's stability can be determined by observing the boundary layer thickness. If it is closer to the boundary layer, the solution is stable (first solution), while if it is further from the boundary layer, the solution is unstable (second solution). As a mathematician, though, proving by mathematical model is a must. Furthermore, even though the second solution is unstable and physically insignificant, it is still a solution to the system of differential equations. Therefore, the goal of this research article is to conduct a stability analysis of the Nandy and Pop [18] dual solutions. It is worth mentioning that the research on stability analysis in thermal radiation has not been thoroughly investigated, and as a result, the present work represents a novel approach.

\section{Methodology}

An electrically conducted incompressible viscous fluid flow at a shrinking surface is considered in a steady over stagnation-point flow in two-dimensional. On the surface, a magnetic field $B_{0}$ of uniform strength is introduced. The stretching/shrinking velocity is assumed to be $u_{w}(x)=c x$ while the external boundary layer velocity is assumed as $U(x)=a x$, with a and c being constant. To be noted, the stretching sheet is denoted by $c>0$, while the shrinking sheet is denoted by $c<0$. For this problem, the steady two-dimensional equations are [18].

$$
\begin{aligned}
& \frac{\partial u}{\partial x}+\frac{\partial v}{\partial y}=0 \\
& u \frac{\partial u}{\partial x}+v \frac{\partial u}{\partial y}=U \frac{d U}{d x}+v \frac{\partial^{2} u}{\partial y^{2}}+\frac{\sigma B_{0}^{2}}{\rho}(U-u) \\
& u \frac{\partial T}{\partial x}+v \frac{\partial T}{\partial y}=\alpha_{m} \frac{\partial^{2} T}{\partial y^{2}}-\frac{1}{\rho_{f} c_{p}} \frac{\partial q_{r}}{\partial y}+\frac{(\rho c)_{p}}{(\rho c)_{f}}\left[\mathrm{D}_{B} \frac{\partial \phi}{\partial y} \frac{\partial T}{\partial y}+\frac{D_{T}}{T_{\infty}}\left(\frac{\partial T}{\partial y}\right)^{2}\right] \\
& u \frac{\partial \phi}{\partial x}+v \frac{\partial \phi}{\partial y}=D_{B} \frac{\partial^{2} \phi}{\partial y^{2}}+\frac{D_{T}}{T_{\infty}} \frac{\partial^{2} T}{\partial y^{2}}
\end{aligned}
$$


and the conditions at boundary are,

$$
\begin{array}{lccrr}
v=0, \quad u=u_{w}(x)=\mathrm{c} x & T=T_{w} \quad \phi=\phi_{w} & \text { at } & y=0 \\
u \rightarrow U(x)=\mathrm{a} x, \quad T \rightarrow T_{\infty}, & \phi \rightarrow \phi_{w} & \text { as } & & y \rightarrow \infty
\end{array}
$$

where $u$ is the $x$-direction velocity and $v$ is the $y$-direction velocity. $T$ is the fluid temperature, $\rho_{f}$ denotes the fluid density, the kinematic viscosity is denoted by $v, \alpha_{m}$ is the thermal diffusivity, $U(x)$ denotes the external fluid's velocity, $\phi$ is the nanoparticle volume fraction, $q_{r}$ is the radiative heat flux, $D_{B}$ is the Brownian diffusion coefficient, $\sigma$ is the fluid's electrical conductivity, $D_{T}$ is the thermophoresis diffusion coefficient, $c_{p}$ denotes constant pressure specific heat and $(\rho c)_{\rho} /(\rho c)_{f}$ is the ratio of the nanofluid material's effective heat capacity to the heat capacity of an ordinary fluid. For thermal radiation, according to the approximation of Rosseland, we obtain [19]

$q_{r}=-\frac{4 \sigma^{*}}{3 k_{1}} \frac{\partial T^{4}}{\partial y}$

where $k_{1}$ is the mean absorption coefficient while $\sigma^{*}$ is the Stefan - Boltzmann constant. $T^{4} \approx 4 T_{\infty}^{3} T-3 T_{\infty}^{4}$ is determined based on the assumption that the temperature variation $T^{4}$ can be enlarged into a Taylor series over $T_{\infty}$ and omitting higher order terms. Eq. (3) can then be reduced to

$u \frac{\partial T}{\partial x}+v \frac{\partial T}{\partial y}=\alpha_{m} \frac{\partial^{2} T}{\partial y^{2}}+\frac{16 \sigma^{*} T_{\infty}^{3}}{3 k_{1} \rho_{f} c_{p}} \frac{\partial^{2} T}{\partial y^{2}}+\frac{(\rho c)_{p}}{(\rho c)_{f}}\left[\mathrm{D}_{B} \frac{\partial \phi}{\partial y} \frac{\partial T}{\partial y}+\frac{D_{T}}{T_{\infty}}\left(\frac{\partial T}{\partial y}\right)^{2}\right]$

Next, we solve Eq. (1) to Eq. (4) using the following similarity variables in combination with the boundary conditions from Eq. (5)

$\psi=\sqrt{a v} x f(\eta), \quad \eta=\sqrt{\frac{a}{v}} y, \quad \theta(\eta)=\frac{T-T_{\infty}}{T_{w}-T_{\infty}}, \quad S(\eta)=\frac{\phi-\phi_{\infty}}{\phi_{w}-\phi_{\infty}}$

where $u=\partial \psi / \partial y, v=-\partial \psi / \partial x$ and $\psi$ is referred to as the stream function. Then,

$u=a x f^{\prime}(\eta)$ and $\quad v=-\sqrt{v a} f(\eta)$

Next, we have obtained the ordinary differential equations shown below by replacing Eq. (8) and Eq. (9) into Eq. (1), Eq. (2), Eq. (4) and Eq. (7).

$$
\begin{aligned}
& f^{\prime \prime \prime}+f f^{\prime \prime}-f^{\prime 2}+1+M\left(1-f^{\prime}\right)=0 \\
& \frac{1}{\operatorname{Pr}}(1+4 \mathrm{R} / 3) \theta^{\prime \prime}+f \theta^{\prime}+N b \theta^{\prime} S^{\prime}+N t \theta^{\prime 2}=0
\end{aligned}
$$


$S^{\prime \prime}+L e f S^{\prime}+\frac{N t}{N b} \theta^{\prime \prime}=0$

where $M=\sigma B_{0}^{2} / \rho_{f} a$ denotes the magnetic parameter, $\operatorname{Pr}=v / \alpha_{m}$ denotes the Prandtl number, $R=4 \delta T_{\infty}^{3} / k_{1} \kappa$ denotes the thermal radiation parameter, $N t=\left((\rho c)_{p} /(\rho \mathrm{c})_{f}\right) D_{T}\left(T_{w}-T_{\infty}\right) / v T_{\infty}$ denotes the thermophoresis parameter, $N b=\left((\rho c)_{p} /(\rho c)_{f}\right) D_{B}\left(\phi_{w}-\phi_{\infty}\right) / v$ denotes the Brownian motion parameter and $L e=v / D_{B}$ is represents the Lewis number. After that, the boundary conditions (5) are simplified to the following:

$f(\eta)=0, \quad f^{\prime}(0)=\alpha=c / a, \quad \theta(0)=1, \quad S(0)=1$

$f^{\prime}(\eta) \rightarrow 1, \quad \theta(\eta) \rightarrow 0, \quad S(\eta) \rightarrow 0$ as $\quad \eta \rightarrow \infty$

with $\alpha$ representing the ratio of stretching/shrinking velocity to the velocity of the free stream. The skin friction coefficient $C_{f}$, the local Nusselt number $N u_{x}$ and the local Sherwood number $S h_{x}$ are defined as

$$
C_{f}=\frac{\mu}{\rho_{f} U^{2}(x)}\left(\frac{\partial u}{\partial y}\right)_{y=0}, \quad N u_{x}=\frac{x}{T_{w}-T_{\infty}}\left(-\frac{\partial T}{\partial y}\right)_{y=0}, \quad S h_{x}=\frac{x}{\left(\phi_{w}-\phi_{\infty}\right)}\left(-\frac{\partial \phi}{\partial y}\right)
$$

We obtain the following by applying variables from Eq. (8)

$$
\operatorname{Re}_{x}^{1 / 2} C_{f}=f^{\prime \prime}(0), \quad \quad \operatorname{Re}_{x}^{-1 / 2} N u_{x}=-\theta^{\prime}(0), \quad S h_{x} \operatorname{Re}_{x}^{-1 / 2}=-S^{\prime}(0)
$$

where $\operatorname{Re}_{x}=U(x) x / v$ denotes the local Reynolds number.

\section{Stability Analysis}

Since the existence of a dual solution, there has been an increase in the number of reports that acknowledge the importance of stability analysis. Firstly, the problem should be regarded as an unsteady problem to commence the stability analysis. Then, Eq. (2) to Eq. (4) come out as

$$
\begin{aligned}
& \frac{\partial u}{\partial t}+u \frac{\partial u}{\partial x}+v \frac{\partial u}{\partial y}=U \frac{d U}{d x}+v \frac{\partial^{2} u}{\partial y^{2}}+\frac{\sigma B_{0}^{2}}{\rho_{f}}(U-u) \\
& \frac{\partial T}{\partial t}+u \frac{\partial T}{\partial x}+v \frac{\partial T}{\partial y}=\alpha_{m} \frac{\partial^{2} T}{\partial y^{2}}-\frac{1}{\rho_{f} c_{p}} \frac{\partial q_{r}}{\partial y}+\frac{(\rho c)_{p}}{(\rho c)_{f}}\left[\mathrm{D}_{B} \frac{\partial \phi}{\partial y} \frac{\partial T}{\partial y}+\frac{D_{T}}{T_{\infty}}\left(\frac{\partial T}{\partial y}\right)^{2}\right] \\
& \frac{\partial \phi}{\partial t}+u \frac{\partial \phi}{\partial x}+v \frac{\partial \phi}{\partial y}=D_{B} \frac{\partial^{2} \phi}{\partial y^{2}}+\frac{D_{T}}{T_{\infty}} \frac{\partial^{2} T}{\partial y^{2}}
\end{aligned}
$$


where $t$ is defined as time. Based on the work of Weidman et al., [10], it is necessary to introduce a time variable with no dimensions, denoted by $\tau$. As a result, for the unsteady problem, we introduced a new dimensionless variable,

$\psi=\sqrt{a v} x f(\eta, \tau), \quad \eta=\sqrt{\frac{a}{v}} y, \quad \theta(\eta, \tau)=\frac{T-T_{\infty}}{T_{w}-T_{\infty}}, \quad S(\eta, \tau)=\frac{\phi-\phi_{\infty}}{\phi_{w}-\phi_{\infty}} \quad \tau=a t$

as a result, Eq. (2) to Eq. (4) can be written in the following way

$$
\begin{aligned}
& \frac{\partial^{3} f}{\partial \eta^{3}}+f \frac{\partial^{2} f}{\partial \eta^{2}}-\left(\frac{\partial f}{\partial \eta}\right)^{2}+1+M\left(1-\frac{\partial f}{\partial \eta}\right)-\frac{\partial^{2} f}{\partial \eta \partial \tau}=0 \\
& \frac{1}{\operatorname{Pr}}\left(1+\frac{4}{3} R\right) \frac{\partial^{2} \theta}{\partial \eta^{2}}+f \frac{\partial \theta}{\partial \eta}+N b \frac{\partial \theta}{\partial \eta} \frac{\partial \phi}{\partial \eta}+N t\left(\frac{\partial \theta}{\partial \eta}\right)^{2}-\frac{\partial \theta}{\partial \tau}=0 \\
& \frac{\partial^{2} \phi}{\partial \eta}+\operatorname{Lef} \frac{\partial \phi}{\partial \eta}+\frac{N t}{N b} \frac{\partial^{2} \theta}{\partial \eta^{2}}-\frac{\partial \phi}{\partial \eta}=0
\end{aligned}
$$

the boundary conditions are followed by the following

$$
\begin{aligned}
& f(0, \tau)=0, \quad \frac{\partial f}{\partial \eta}(\eta, \tau)=\alpha, \quad \theta(0, \tau)=1, \quad S(0)=1 \\
& \frac{\partial f}{\partial \eta}(\eta, \tau) \rightarrow 1, \quad \theta(\eta, \tau) \rightarrow 0, \quad S(\eta, \tau) \rightarrow 0 \quad \text { as } \quad \eta \rightarrow \infty
\end{aligned}
$$

To ascertain the steady flow solution's stability, $f(\eta)=f_{0}(\eta), \theta(\eta)=\theta_{0}(\eta)$ and $S(\eta)=S_{0}(\eta)$ satisfy the boundary - value problem (1)-(5) [6-17]

$$
\begin{aligned}
& f(\eta, \tau)=f_{0}(\eta)+e^{-\gamma \tau} F(\eta, \tau) \\
& \theta(\eta, \tau)=\theta_{0}(\eta)+e^{-\gamma \tau} T(\eta, \tau) \\
& S(\eta, \tau)=S_{0}(\eta)+e^{-\gamma \tau} G(\eta, \tau)
\end{aligned}
$$

where $F(\eta, \tau)$ is small relative to $f_{0}(\eta), T(\eta, \tau)$ is small relative to $\theta_{0}(\eta), G(\eta, \tau)$ is small relative to $S_{0}(\eta)$ and $\gamma$ is an is an unidentified eigenvalue. By replacing (24) in (20) - (22), the following result is obtained:

$$
\frac{\partial^{3} F}{\partial \eta^{3}}+f_{0} \frac{\partial^{2} F}{\partial \eta^{2}}+f_{0}^{\prime \prime} F+\left(\gamma-M-2 f_{0}^{\prime}\right) \frac{\partial F}{\partial \eta}-\frac{\partial^{2} F}{\partial \eta \partial \tau}=0
$$




$$
\begin{aligned}
& \frac{1}{\operatorname{Pr}}\left(1+\frac{4}{3} R\right) \frac{\partial^{2} T}{\partial \eta}+\left(f_{0}+N b S_{0}+2 N t \theta_{0}^{\prime}\right) \frac{\partial T}{\partial \eta}+\theta_{0}^{\prime} F+N b \theta_{0}^{\prime} \frac{\partial G}{\partial \eta}+\gamma T-\frac{\partial \theta}{\partial \tau}=0 \\
& \frac{\partial^{2} G}{\partial \eta^{2}}+L e f_{0} \frac{\partial G}{\partial \eta}+L e S_{0}^{\prime} F+\frac{N t}{N b} \frac{\partial^{2} T}{\partial \eta^{2}}+\gamma G-\frac{\partial S}{\partial \tau}=0
\end{aligned}
$$

subsequently, boundary conditions

$$
\begin{aligned}
& F(0, \tau)=0, \quad \frac{\partial F}{\partial \eta}(0, \tau)=0, \quad T(0, \tau)=0, \quad G(0, \tau)=0 \\
& \frac{\partial F}{\partial \eta}(\eta, \tau) \rightarrow 0, \quad T(\eta, \tau) \rightarrow 0 \quad G(\eta, \tau) \rightarrow 0 \quad \text { as } \quad \eta \rightarrow \infty
\end{aligned}
$$

By setting $\tau=0$, the steady solutions (10) - (12) obtain the solutions $f(\eta)=f_{0}(\eta), \theta(\eta)=\theta_{0}(\eta)$ and $S(\eta)=S_{0}(\eta)$. Therefore, $F=F_{0}(\eta), T=T_{0}(\eta)$ and $G=G_{0}(\eta)$ in (25) - (27) identify the initial increment of the solution (24). Then, the equations reduce into

$$
\begin{aligned}
& F_{0}^{\prime \prime \prime}+f_{0} F_{0}^{\prime \prime}+f_{0}^{\prime \prime} F_{0}+\left(\gamma-M^{2}-2 f_{0}^{\prime}\right) F_{0}^{\prime}=0 \\
& \frac{1}{P r}\left(1+\frac{4}{3} R\right) T_{0}^{\prime \prime}+\left(f_{0}+N b S_{0}^{\prime}+2 N t \theta_{0}^{\prime}\right) T_{0}^{\prime}+\theta_{0}^{\prime} F_{0}^{\prime}+N b \theta_{0}^{\prime} G_{0}^{\prime}+\gamma T_{0}=0 \\
& G_{0}^{\prime \prime}+\operatorname{Lef}_{0} G_{0}^{\prime}+L e S_{0} F_{0}+\frac{N b}{N t} T_{0}^{\prime \prime}+\gamma G_{0}=0
\end{aligned}
$$

and the homogeneous boundary conditions are

$$
\begin{aligned}
& F_{0}(0)=0, \quad F_{0}^{\prime}(0)=0, \quad T_{0}(0)=0, \quad G_{0}(0)=0 \\
& F_{0}^{\prime}(\eta) \rightarrow 0, \quad T_{0}(0) \rightarrow 0, \quad G_{0}(\eta) \rightarrow 0 \quad \text { as } \quad \eta \rightarrow \infty
\end{aligned}
$$

It is essential to mention that the stability of the steady flow solutions for $f_{0}(\eta), \theta_{0}(\eta)$ and $S_{0}(\eta)$ is evaluated by the smallest eigenvalue $\gamma$ for a set of parameters with specific values, like $M, s, P r$, $N b, N t, R$, and Le. Then, to define the range of possible eigenvalues, we relax a boundary condition on $F_{0}(\eta), T_{0}(0)$ or $G_{0}(\eta)$ [8]. The boundary condition $F_{0}^{\prime} \rightarrow 0$ as $\eta \rightarrow \infty$ has been chosen to be relaxed for this study. Following that, we solved the Eq. (29) to Eq. (31) using the update boundary condition, which was denoted by $F_{0}^{\prime \prime}(0)=1$.

\section{Finding and Discussion}

Mathematical solutions for the governing ODEs (10) - (12) and boundary conditions (13) are obtained by numerically solving them with the bvp4c solver in Matlab. The dual solutions are 
discovered by making guesses about the numerous initial values for $f^{\prime \prime}(0),-\theta^{\prime}(0)$ and $-S^{\prime}(0)$, and all profiles meet the boundary condition (13). The numerical findings reported in Table 1 are consistent with Nandy and Pop's [18] previous work. The solution is unique in this study if $\alpha>-1.0$, a dual solution for $\alpha_{c}<\alpha \leq-1$ and there is no solution for $\alpha<\alpha_{c}$ where $\alpha_{c}$ denotes the critical value of $\alpha$.

\section{Table 1}

Comparison values of $-\theta^{\prime}(0)$ when $\alpha=-1.20, \operatorname{Pr}=0.71, L e=1.0$ and $R=0.1$

\begin{tabular}{llllllll}
\hline $\mathrm{M}$ & $\mathrm{Nt}$ & \multicolumn{2}{c}{ Nandy and Pop [18] } & \multicolumn{5}{l}{ Present results } \\
& & $\mathrm{Nb}=0.1$ & 0.3 & 0.5 & $\mathrm{Nb}=0.1$ & 0.3 & 0.5 \\
\hline 0.1 & 0.1 & 0.154170 & 0.144231 & 0.134726 & 0.1541709 & 0.1442309 & 0.1347271 \\
& 0.3 & 0.145413 & 0.135998 & 0.127002 & 0.1454135 & 0.1360014 & 0.1270085 \\
& 0.5 & 0.137219 & 0.128307 & 0.119798 & 0.1372205 & 0.1283085 & 0.1197988 \\
0.2 & 0.1 & 0.176443 & 0.164952 & 0.153983 & 0.1764439 & 0.1649564 & 0.1539852 \\
& 0.3 & 0.166597 & 0.155712 & 0.145304 & 0.1665970 & 0.1557016 & 0.1453039 \\
& 0.5 & 0.157369 & 0.147035 & 0.137181 & 0.1573698 & 0.1470370 & 0.1371829 \\
0.3 & 0.1 & 0.193236 & 0.180578 & 0.168485 & 0.1932395 & 0.1805732 & 0.1684853 \\
& 0.3 & 0.182591 & 0.170563 & 0.159094 & 0.1825913 & 0.1705649 & 0.1590968 \\
& 0.5 & 0.172602 & 0.161184 & 0.150296 & 0.1726028 & 0.1611850 & 0.1503053 \\
\hline
\end{tabular}

Figure 1 to Figure 3 depict the profile of velocity, temperature, and concentration. The appointed value of $\alpha$ resulted in non-unique solutions, as shown in the figures, which is supported by the obtained results by Nandy and Pop [18]. For the second solution, the boundary layer is thinner while for the first solution, it is thicker. This situation is valid for all profiles. A consequence of this is that it is expected that the first solution will be both physically significant and stable, whereas the second solution will be both unstable and physically insignificant. Additionally, all profiles demonstrate asymptotically satisfying the boundary conditions (13) as $f^{\prime}(\eta) \rightarrow 1, \theta(\eta) \rightarrow 0$ and $S(\eta) \rightarrow 0$.

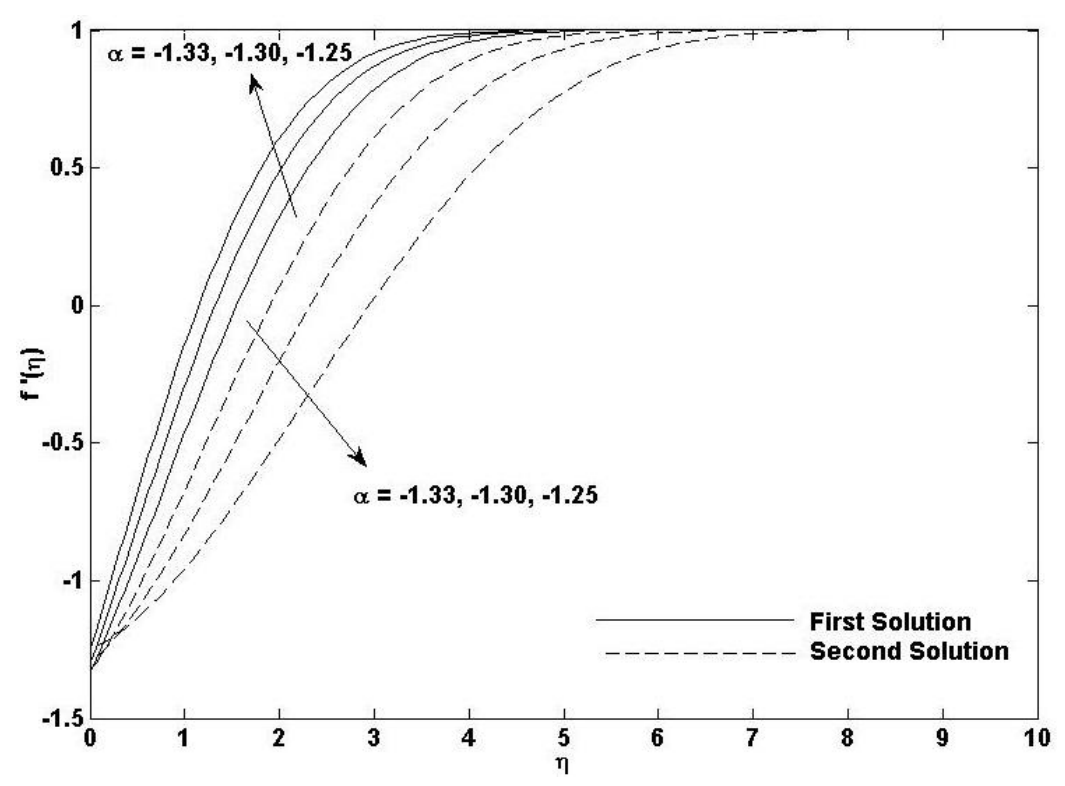

Fig. 1. Velocity profiles for selected values of $\alpha$ 


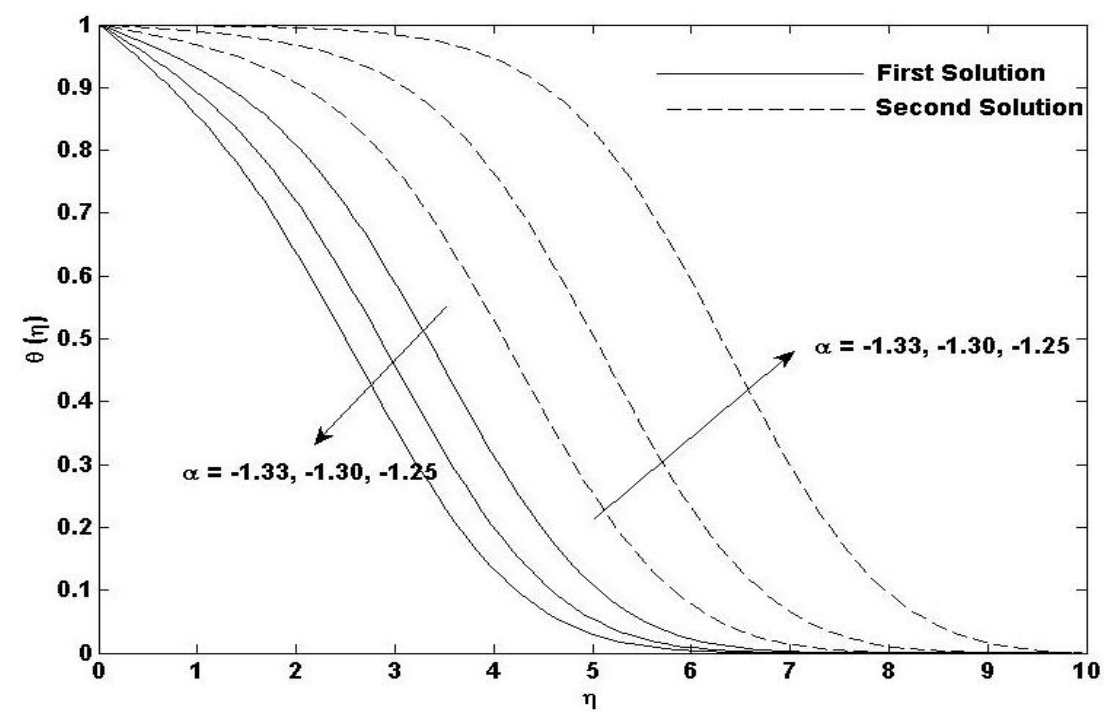

Fig. 2. Temperature profiles for different values of $\alpha$

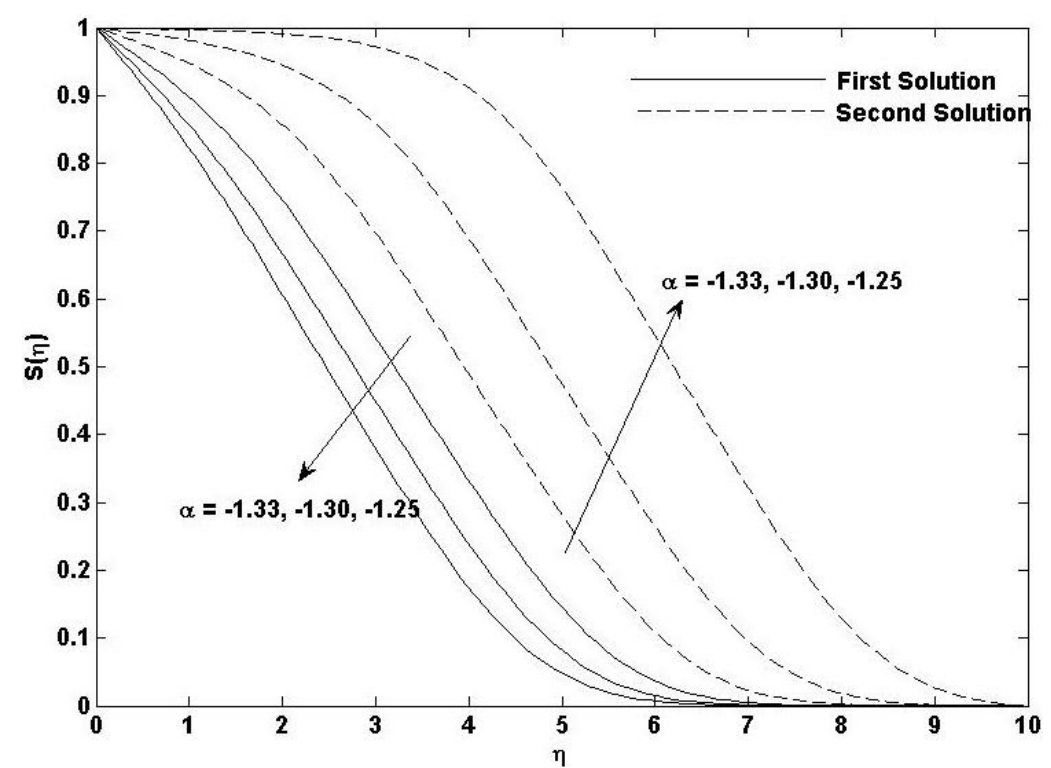

Fig. 3. Concentration profiles for selected values of $\alpha$

The smallest eigenvalue $\gamma$ is shown in Table 2 for various values of $\alpha$ with $R=0,0.2$. As previously stated, if the smallest eigenvalue yields a positive result, then the flow will be considered stable. As can be seen, the upper branch's $\gamma$ value is real and positive, while the lower branch's $\gamma$ value is real and negative. Therefore, the first solution exhibits linear stability, whereas the second solution exhibits linear instability. Under this situation, only the upper branch is physically considerable, while the lower branch wasn't. 
Table 2

Smallest eigenvalues of $\gamma$ with selected values of $\alpha$ and $M=0.1$

\begin{tabular}{llll}
\hline$R$ & $\alpha$ & Upper Branch & Lower Branch \\
\hline 0 & -1.33 & 0.18422 & -0.17788 \\
& -1.30 & 0.50216 & -0.45640 \\
& -1.25 & 0.79501 & -0.68089 \\
0.2 & -1.20 & 1.01066 & -0.82430 \\
& -1.31 & 0.42210 & -0.38958 \\
& -1.29 & 0.57183 & -0.51265 \\
& -1.27 & 0.69177 & -0.60540 \\
\hline
\end{tabular}

\section{Conclusions}

The stability analysis is examined for the flow at the stagnation point as well as heat transfer in nanofluid with the effect of thermal radiation and magnetic field on the shrinking sheet. This study contributed by confirming that, based on the results of a stability analysis, linear stability exists in the upper branch whereas linear instability exists in the other branch. This statement is proved by the finding that the upper branch has a positive value of $\gamma$ whereas the lower branch has a negative value of $\gamma$.

\section{Acknowledgments}

Universiti Malaysia Sarawak, Universiti Putra Malaysia, and the Malaysian Ministry of Education (MyBrain) provided financial support to the writers (MyBrain).

\section{References}

[1] Oo, Ye Min, Makatar Wae-hayee, and Chayut Nuntadusit. "Experimental and Numerical Study on the Effect of Teardrop Dimple/Protrusion Spacing on Flow Structure and Heat Transfer Characteristics." Journal of Advanced Research in Experimental Fluid Mechanics and Heat Transfer 2, no. 1 (2020): 17-32.

[2] Idris, Muhammad Syafiq, Irnie Azlin Zakaria, and Wan Azmi Wan Hamzah. "Heat Transfer and Pressure Drop of Water Based Hybrid Al2O3: SiO2 Nanofluids in Cooling Plate of PEMFC." Journal of Advanced Research in Numerical Heat Transfer 4, no. 1 (2021): 1-13. https://doi.org/10.37934/arfmts.88.3.96109

[3] Jahan, Sultana, M. Ferdows, M. D. Shamshuddin, and Khairy Zaimi. "Effects of Solar Radiation and Viscous Dissipation on Mixed Convective Non-Isothermal Hybrid Nanofluid over Moving Thin Needle." Journal of Advanced Research in Micro and Nano Engineering 3, no. 1 (2021): 1-11.

[4] Pasha, Amjad Ali, Meshal Nuwaym Al-Harbi, Surfarazhussain S. Halkarni, Nazrul Islam, D. Siva Krishna Reddy, S. Nadaraja Pillai, and Ufaith Qadiri. "CFD study of Convective Heat Transfer of Water Flow Through Micro-Pipe with Mixed Constant Wall Temperature and Heat Flux Wall Boundary Conditions." CFD Letters 13, no. 7 (2021): 13-26. https://doi.org/10.37934/cfdl.13.7.1326

[5] Halim, Nur Fazlin Che, and Nor Azwadi Che Sidik. "Nanorefrigerants: A Review on Thermophysical Properties and Their Heat Transfer Performance." Journal of Advanced Research in Applied Sciences and Engineering Technology 20, no. 1 (2020): 42-50. https://doi.org/10.37934/araset.20.1.4250

[6] Merkin, J. H. "On dual solutions occurring in mixed convection in a porous medium." Journal of Engineering Mathematics 20, no. 2 (1986): 171-179. https://doi.org/10.1007/BF00042775

[7] Merrill, Keith, Matthew Beauchesne, Joseph Previte, Joseph Paullet, and Patrick Weidman. "Final steady flow near a stagnation point on a vertical surface in a porous medium." International Journal of Heat and Mass Transfer 49, no. 23-24 (2006): 4681-4686. https://doi.org/10.1016/j.ijheatmasstransfer.2006.02.056

[8] Harris, S. D., D. B. Ingham, and I. Pop. "Mixed convection boundary-layer flow near the stagnation point on a vertical surface in a porous medium: Brinkman model with slip." Transport in Porous Media 77, no. 2 (2009): $267-285$. https://doi.org/10.1007/s11242-008-9309-6

[9] Roşca, Alin V., and loan Pop. "Flow and heat transfer over a vertical permeable stretching/shrinking sheet with a second order slip." International Journal of Heat and Mass Transfer 60 (2013): $355-364$. https://doi.org/10.1016/j.ijheatmasstransfer.2012.12.028 
[10] Weidman, P. D., D. G. Kubitschek, and A. M. J. Davis. "The effect of transpiration on self-similar boundary layer flow over moving surfaces." International Journal of Engineering Science 44, no. 11-12 (2006): $730-737$. https://doi.org/10.1016/j.ijengsci.2006.04.005

[11] Postelnicu, A., and loan Pop. "Falkner-Skan boundary layer flow of a power-law fluid past a stretching wedge." Applied Mathematics and Computation 217, no. 9 (2011): 4359-4368. https://doi.org/10.1016/j.amc.2010.09.037

[12] Ishak, Anuar. "Flow and heat transfer over a shrinking sheet: A stability analysis." International Journal of Mathematical and Computational Sciences 8, no. 5 (2014): 902-906.

[13] Ishak, Anuar. "Dual solutions in mixed convection boundary layer flow: A stability analysis." International Journal of Mathematical and Computational Sciences 8, no. 9 (2014): 1216-1219.

[14] Ismail, Nurul Syuhada, Norihan Md Arifin, Norfifah Bachok, and Norhasimah Mahiddin. "Flow and heat transfer on a moving flat plate in a parallel stream with constant surface heat flux: A stability analysis." Indian Journal of Science and Technology 9, no. 31 (2016). https://doi.org/10.17485/ijst/2016/v9i31/97732

[15] Ismail, N. S., N. M. Arifin, R. Nazar, N. Bachok, and N. Mahiddin. "Stability analysis in stagnation-point flow towards a shrinking sheet with homogeneous-Heterogeneous reactions and suction effects." International Journal of Applied Engineering Research 11, no. 18 (2016): 9229-9235. https://doi.org/10.1063/1.4972153

[16] Ismail, N. S., N. M. Arifin, and R. Nazar. "The stagnation-point flow and heat transfer of nanofluid over a shrinking surface in magnetic field and thermal radiation with slip effects: a stability analysis." In Journal of Physics: Conference Series, vol. 890, no. 1, p. 012055. IOP Publishing, 2017. https://doi.org/10.1088/1742$6596 / 890 / 1 / 012055$

[17] Ismail, Nurul Syuhada, Norihan Md Arifin, Norfifah Bachok, and Norhasimah Mahiddin. "The stagnation-point flow towards a shrinking sheet with homogeneous-heterogeneous reactions effects: A stability analysis." In AIP Conference Proceedings, vol. 1795, no. 1, p. 020009. AIP Publishing LLC, 2017. https://doi.org/10.1063/1.4972153

[18] Nandy, Samir Kumar, and loan Pop. "Effects of magnetic field and thermal radiation on stagnation flow and heat transfer of nanofluid over a shrinking surface." International Communications in Heat and Mass Transfer 53 (2014): 50-55. https://doi.org/10.1016/i.icheatmasstransfer.2014.02.010

[19] Brewster, M. Quinn. Thermal radiative transfer and properties. John Wiley \& Sons, 1992. 